\title{
QUALITY IMPROVEMENT Evaluation of START (STrAtegies for RelaTives) adapted for carers of people with Lewy body
} dementia

\author{
Authors: Jennifer A Foley, ${ }^{\mathrm{A}}$ Charlotte Dore, ${ }^{\mathrm{B}}$ Angeliki Zarkali, ${ }^{\mathrm{C}}$ Gill Livingston, ${ }^{\mathrm{D}}$ Lisa Cipolotti, \\ Catherine J Mummery ${ }^{\mathrm{F}}$ and Rimona S Weil ${ }^{\mathrm{F}}$
}

Family carers of people with Lewy body dementia (LBD) have a particularly high burden of care, as LBD has a faster rate of decline, greater physical dependence and additional neuropsychiatric disturbances compared with other dementias. Despite this, there are no evidence-based support services designed specifically for LBD carers. STrAtegies for RelaTives (START) is an eight-session, individually delivered coping therapy that has been shown in a randomised controlled trial to reduce depression and anxiety symptoms and increase quality of life in carers of people with dementia, with effects lasting several years. We adapted START for LBD, and piloted its use both face-to-face and on the phone with 10 carers to test acceptability and indications of similar effects in this group. Our findings suggest that the therapy was acceptable and feasible using either delivery mode, providing much appreciated and needed strategies, education and support for carers of people with LBD. Trials of effectiveness are now needed.

KEYWORDS: Lewy body dementia, family carers, quality of life, support

DOI: $10.7861 /$ fhj.2020-0003

\section{Introduction}

Lewy body dementia (LBD) is a common cause of dementia, ${ }^{1}$ characterised by progressive loss of intellectual function that occurs either alongside new-onset parkinsonism (dementia with

Authors: ${ }^{A}$ clinical neuropsychologist, National Hospital for Neurology and Neurosurgery, London, UK and University College London Institute of Neurology, London, UK; ${ }^{B}$ assistant psychologist, National Hospital for Neurology and Neurosurgery, London, UK; ${ }^{C}$ clinical research fellow, University College London, London, UK;

${ }^{D}$ professor of psychiatry of older people, University College London, London, UK and Camden and Islington NHS Foundation Trust, London, UK; Ehead of neuropsychology, National Hospital for Neurology and Neurosurgery, London, UK; F consultant neurologist, University College London, London, UK
Lewy bodies; DLB) or within the context of established Parkinson's disease (Parkinson's disease dementia; PDD). Unlike Alzheimer's disease, cognitive symptoms typically present as difficulties with visuospatial and executive functioning rather than episodic memory, and demonstrate a more rapid rate of decline. ${ }^{2}$

Studies consistently demonstrate that carer burden in LBD is higher and more varied than in other dementias. ${ }^{3,4}$ Cognitive symptoms are accompanied by early disturbing neuropsychiatric features of hallucinations and delusions, as well as challenging behavioural issues of apathy, agitation, anxiety and depression. ${ }^{5}$ In addition, LBD is associated with higher levels of frailty and physical dependence than other dementias, necessitating greater levels of physical and personal care, pharmacotherapy and community services, and support for almost all activities of daily living. ${ }^{6}$ Most of this is delivered by family carers, typically spouses, who on average provide care for over 120 hours per week. ${ }^{7}$

As a consequence, LBD carers endure high levels of chronic stress, with associated substantial costs to their own mood, health and finances. ${ }^{8}$ They report social isolation, feeling estranged from both the patient and their wider social network. ${ }^{9}$ Both patient and carer may become stigmatised and feared, with few understanding the symptoms of LBD, particularly the neuropsychiatric features, or the considerable carer burden. ${ }^{10}$ LBD carers also report feeling insufficiently supported by medical professionals. They often struggle to have the symptoms understood or diagnosed, and even when a diagnosis is made, they report a lack of available information or support, as well as difficulty accessing specialists. ${ }^{8}$ Unsurprisingly, these factors increase the risk of anger and aggression towards the patient and of deterioration in patients' symptoms, and hasten transfer to formal care. Although the National Institute for Health and Care Excellence (NICE) recommends the provision of emotional and psychological support to carers of people living with dementia, there are still few support services available and none designed specifically for the unique challenges faced by LBD carers. ${ }^{11}$ Therefore, there is a clear and unmet need for strategies to improve support for carers of people with this common and debilitating form of dementia.

\section{Intervention}

STrAtegies for RelaTives (START) is an 8-week, manualised, individual psychological intervention designed for carers of people with dementia. ${ }^{12}$ It includes psychoeducation about dementia, 
challenging behaviour, communication, relaxation and planning for the future, provides techniques for behaviour management and focuses on self-care. It has been shown to reduce anxiety and depression with effects lasting up to 6 years, incurring a mean cost of $£ 253$ per carer, later offset by reduced use of health and social care services. ${ }^{13}$ As START was designed for dementia in general, it focuses on cognitive symptoms of episodic memory impairment and generic aspects of cognitive decline rather than on the issues specific to LBD. Therefore, in this study, we adapted START to the particular challenges of LBD and sought to examine its feasibility and acceptability in a small sample of LBD carers.

We adapted START manuals by modifying the vignettes and psychoeducation to focus on the cognitive and neuropsychiatric symptoms characteristic of LBD, rather than episodic memory impairment. We also revised the discussion of specialist care services to reflect the specific neuropsychiatric and neurological services involved in the management of LBD. We piloted the use of START in a clinical service for LBD carers for 2.5 months using local funding. A psychology graduate was appointed, trained and supervised by a clinical neuropsychologist. In light of LBD carers' increased burden, and in contrast with the original START programme, we also offered START over the telephone as an alternative to the carer having to leave the patient to attend our centre. Apart from this and the minor changes described above, the core elements of the START programme were unchanged. See Box 1 for session topics.

We collected demographic information and measured quality of life, depression and anxiety at the start and end of the programme. We measured quality of life using the Adult Carer Quality of Life Questionnaire (AC-QoL), which is designed for carers and previously used in LBD. ${ }^{14}$ This measure comprises eight subscales evaluating unpaid carers' perceptions of support for caring (the extent to which carers perceive feeling supported); caring choice (the extent to which carers feel they have control over their own life); caring stress (the mental and physical stress from caring); money matters (how carers feel about their finances); personal growth (the extent to which carers feel they have undergone personal growth as a function of the caring); sense of value (the extent to which the carer feels valued in their role); ability to care; and carer satisfaction. We measured depression and anxiety using the Hospital Anxiety and Depression Scale (HADS), which has previously been used to measure emotional distress in dementia carers. ${ }^{15}$ Carers also gave written qualitative feedback of their experience of the service, from which we extracted themes using a grounded theory approach.

\section{Box 1. STrAtegies for RelaTives for Lewy body} dementia session topics

Education about Lewy body dementia, carer stress and relaxation Managing problem behaviours: trigger-behaviour-reaction Managing problem behaviours: developing a behaviour plan Identifying and changing unhelpful thoughts

Communication skills

Planning for the future and accessing practical support

Increasing pleasant activities

Maintaining skills learnt over time

\section{Results}

During the 2.5 months, 21 carers were referred to the service. The first 10 were offered START and accepted. These were all full-time carers of their partners with DLB (six; $60 \%$ ) or PDD (four; $40 \%$ ). On the AC-QoL, they reported quality of life in the mid-range for all subscales except for caring choice, which was low (4.7/15). On the HADS, all carers scored in the clinical range for either anxiety or depression, with means in the moderate levels (anxiety: 12.8/21; depression: 11.0/21). Five carers chose to have all sessions by telephone, four carers chose to have them in person, and one chose a combination of both. Notably, each carer completed all of the eight sessions, with an attrition rate of zero. At the end of therapy, the carers endorsed mid-range quality of life on all subscales of the AC-QoL, including caring choice (5.9/15), and support for caring was improved after the programme (mean $7.2 \pm 3.5$ before; mean $8.8 \pm 4.0$ after; $t=2.4 ; p=0.04$ ). On the HADS, anxiety remained in the moderate level (11.5/21), but depression had reduced to below clinical cut-off (7.5/21). Written feedback revealed themes of appreciating having their difficulties recognised, enjoying being able to share concerns, improved confidence in their ability to care and feeling more prepared for the future.

In our study, LBD carers demonstrated low quality of life and high rates of emotional distress. When we evaluated agreement to the therapy, attendance at sessions and written feedback, our findings suggested that our modified version of START, adapted for LBD, was both feasible and acceptable to our LBD carers. Indeed, all those offered START accepted and completed the programme, illustrating the need and desire for a psychological support service designed specifically for LBD carers. This high adherence rate may also have been in part because we offered the therapy by telephone, permitting carers to remain with the LBD patient. Written feedback confirmed that carers felt more supported and confident about coping with the disease. Although our sample size limited quantitative analysis, the questionnaire scores provide preliminary evidence that participation in this modified version of START may be associated with a reduction in emotional distress and improvement in quality of life.

\section{Conclusion}

Our small study suggests that the psychological intervention of START, when adapted for LBD, is both acceptable and feasible for carers of people with LBD and may offer important support to those coping with the relentless and substantial challenges posed by this specific dementia. Larger and controlled trials of effectiveness are now needed, but the potential of using phonedelivered support in this population suggests that costs per carer may be significantly reduced.

\section{Funding}

This programme was supported by a Small Acorns Grant from the National Brain Appeal. Rimona S Weil is supported by a fellowship from the Wellcome Trust. Angeliki Zarkali is supported by a fellowship from Alzheimer's Research UK. Gill Livingston, Lisa Cipolotti and Catherine ] Mummery are supported by the UCLH National Institute for Health Research (NIHR) Biomedical Research Centre. Gill Livingston also received funding from the NIHR Applied Health Care (ARC) North Thames at Barts Health NHS Trust. 


\section{Conflicts of interest}

Rimona S Weil has received personal fees from GE Healthcare.

\section{References}

1 Walker Z, Possin KL, Boeve BF, Aarsland D. Lewy body dementias. Lancet 2015;386:1683-97.

2 Price A, Farooq R, Yuan J-M, Menon VB, Cardinal RN, O'Brien JT. Mortality in dementia with Lewy bodies compared with Alzheimer's dementia: a retrospective naturalistic cohort study. BMJ Open 2017;7:e017504

3 Zweig YR, Galvin JE. Lewy body dementia: the impact on patients and caregivers. Alzheimers Res Ther 2014;6:21.

4 Svendsboe E, Terum T, Testad I et al. Caregiver burden in family carers of people with dementia with Lewy bodies and Alzheimer's disease. Int J Geriatr Psychiatry 2016;31:1075-83.

5 McKeith IG, Boeve BF, Dickson DW et al. Diagnosis and management of dementia with Lewy bodies: Fourth consensus report of the DLB Consortium. Neurology 2017:89:88-100.

6 Mueller C, Ballard C, Corbett A, Aarsland D. The prognosis of dementia with Lewy bodies. Lancet Neurol 2017;16:390-8.

7 Lawson RA, Yarnall AJ, Johnston F et al. Cognitive impairment in Parkinson's disease: impact on quality of life of carers. Int J Geriatr Psychiatry 2017;32:1362-70.

8 Tan SB, Williams AF, Morris ME. Experiences of caregivers of people with Parkinson's disease in Singapore: A qualitative analysis. J Clin Nurs 2012;21:2235-46.

9 Vatter S, McDonald KR, Stanmore E et al. A qualitative study of female caregiving spouses' experiences of intimate relationships as cognition declines in Parkinson's disease. Age Ageing 2018;47: 604-10.
10 Galvin JE, Duda JE, Kaufer DI et al. Lewy body dementia: the caregiver experience of clinical care. Parkinsonism Relat Disord 2010;16:388-92.

11 National Institute for Health and Care Excellence. Dementia: assessment, management and support for people living with dementia and their carers: NICE guideline [NG97]. NICE, 2018. www.nice.org.uk/guidance/ng97 [Accessed 07 September 2020].

12 Livingston G, Barber J, Rapaport P et al. Clinical effectiveness of a manual based coping strategy (START, STrAtegies for RelaTives) in promoting the mental health of carers of family members with dementia: pragmatic randomised controlled trial. BMJ 2013; 347:f6276

13 Livingston G, Manela M, O'Keeffe A et al. Clinical effectiveness of the START (STrAtegies for RelaTives) psychological intervention for family carers and the effects on the cost of care for people with dementia: 6-year follow-up of a randomised controlled trial. $\mathrm{Br}$ ] Psychiatry 2020;216:35-42.

14 Elwick H, Joseph S, Becker S, Becker F. Manual for the Adult Carer Quality of Life Questionnaire (AC-QoL). London: The Princess Royal Trust for Carers, 2010. www.researchgate.net/publication/237279984_Manual_for_the_Adult_Carer_Quality_of_ Life_Questionnaire_AC-QoL/link/0c96051bb1183e639a000000/ download [Accessed 07 September 2020].

15 Zigmond AS, Snaith RP. The Hospital Anxiety and Depression Scale. Acta Psychiatr Scand 1983;67:361-70.

Address for correspondence: Dr Jennifer A Foley, Department of Neuropsychology, Box 37, National Hospital for Neurology and Neurosurgery, Queen Square, London WC1N 3BG, UK. Email: jennifer.foley@nhs.net 